\title{
ON ORTHOGONAL POLYNOMIALS WITH RESPECT TO VARYING MEASURES ON THE UNIT CIRCLE
}

\author{
K. PAN
}

\begin{abstract}
Let $\left\{\phi_{n}(d \mu)\right\}$ be a system of orthonormal polynomials on the unit circle with respect to $d \mu$ and $\left\{\psi_{n, m}(d \mu)\right\}$ be a system of orthonormal polynomials on the unit circle with respect to the varying measures $d \mu /\left|w_{n}(z)\right|^{2}, z=$ $e^{i \theta}$, where $\left\{w_{n}(z)\right\}$ is a sequence of polynomials, $\operatorname{deg} w_{n}=n$, whose zeros $w_{n, 1}, \ldots, w_{n, n}$ lie in $|z|<1$ The asymptotic behavior of the ratio of the two systems on and outside the unit circle is obtained.
\end{abstract}

\section{INTRODUCTION}

Let $d \mu$ be a finite positive Borel measure with an infinite set as its support on $[0,2 \pi]$. We denote $\mu^{\prime}(\theta)=d \mu / d \theta$, the Radon-Nikodým derivative of $d \mu$ with respect to $d \theta$. The Szegö polynomial with respect to $d \mu$ is denoted by $\phi_{n}(d \mu), n=0,1,2, \ldots$, i.e., $\phi_{n}(d \mu, z)=\phi_{n}(z):=\kappa_{n}(d \mu) z^{n}+$ lower degree terms, $\kappa_{n}(d \mu)>0$, and

$$
\frac{1}{2 \pi} \int_{0}^{2 \pi} \phi_{n}(d \mu, z) \overline{\phi_{m}(d \mu, z)} d \mu(\theta)=\delta_{n, m}, \quad z=e^{i \theta},
$$

for $m, n=0,1,2, \ldots$.

Let $\left\{w_{n}(z)\right\}_{n=1}^{\infty}$ be a sequence of polynomials such that for each $n \in \mathbf{N}$, $w_{n}(z)$ has degree $n$ and all its zeros $w_{n, 1}, \ldots, w_{n, n}$ lie in $|z|<1$. For each $n$, we can define the orthonormal polynomials $\psi_{n, m}(z)=\alpha_{n, m} z^{m}+\cdots$, with respect to the varying measure $d \mu /\left|w_{n}(z)\right|^{2}$, that are uniquely determined by the conditions

$$
\begin{gathered}
\frac{1}{2 \pi} \int_{0}^{2 \pi} \psi_{n, m}(z) z^{-j} \frac{d \mu(\theta)}{\left|w_{n}(z)\right|^{2}}=0, \quad j=0, \ldots, m-1, \\
\frac{1}{2 \pi} \int_{0}^{2 \pi}\left|\psi_{n, m}(z)\right|^{2} \frac{d \mu(\theta)}{\left|w_{n}(z)\right|^{2}}=1, \quad \text { and } \quad \alpha_{n, m}>0, \quad z=e^{i \theta} .
\end{gathered}
$$

If $h$ is a nonnegative measurable function on $[0,2 \pi]$ such that $\log h \in L^{1}$, then the Szegö function $D(h)$ is defined by

$$
D(h, z)=\exp \left(\frac{1}{4 \pi} \int_{0}^{2 \pi} \log h(t) \frac{u+z}{u-z} d t\right), \quad u=e^{i t} .
$$

Received by the editors December 29, 1993 and, in revised form, May 17, 1994.

1991 Mathematics Subject Classification. Primary 42C05.

Key words and phrases. Orthogonal polynomials, asymptotic properties. 
We say that $h$ satisfies the Szegö condition if $D(h, 0) \neq 0$. It is well known that $D(h) \in H^{2}$; and for almost every $t \in[0,2 \pi]$

$$
\lim _{r \nearrow 1} D\left(h, r e^{i t}\right)=D\left(h, e^{i t}\right)
$$

exists and

$$
\left|D\left(h, e^{i t}\right)\right|^{2}=h(t)
$$

for almost every $t \in[0,2 \pi]$.

Let $\mathscr{P}_{n}$ be the set of all polynomials of degree at most $n$. For each polynomial $p_{n}(z)$ of degree $n$, we define $p_{n}^{*}(z)=z^{n} \overline{p_{n}(1 / \bar{z})}$. Then it is easy to see that $\left|p_{n}^{*}(z)\right|=\left|p_{n}(z)\right|$ for $|z|=1$.

Szegö's theory is concerned with the asymptotic behavior of $\phi_{n}(d \mu, z)$. One can state the main theorem as follows:

$$
\lim _{n \rightarrow \infty} \phi_{n}^{*}(z)=\frac{1}{D\left(\mu^{\prime}, z\right)}
$$

uniformly for $|z| \leq s<1$ whenever $\log \mu^{\prime} \in L^{1}$ (cf. [G]).

Recently, the orthonormal polynomials $\psi_{n, m}$ have been studied in rational interpolation with free poles and with prescribed poles. As in the theory of Szegö polynomials, convergence results and asymptotic and interpolation properties have been studied (cf. [Lo1-4] and [P2]). The relations between $\psi_{n, m}(z)$ and the orthonormal rational functions with prescribed poles have been studied as well (cf. [P1] and [LP]). A comparison with $\phi_{n}(z)$ needs further study.

The following result, analogous to (1.1), holds:

$$
\lim _{n \rightarrow \infty} \frac{\psi_{n, n+k}^{*}(z)}{w_{n}^{*}(z)}=\frac{1}{D\left(\mu^{\prime}, z\right)}
$$

uniformly for $|z| \leq s<1$ whenever $\log \mu^{\prime} \in L^{1}$ and

$$
\lim _{n \rightarrow \infty} \sum_{m=1}^{n}\left(1-\left|w_{n, m}\right|\right)=\infty
$$

where $k$ is a fixed integer (cf. [Lol]).

Thus if $\log \mu^{\prime} \in L^{1}$ and $\lim _{n \rightarrow \infty} \sum_{m=1}^{n}\left(1-\left|w_{n, m}\right|\right)=\infty$, then we have

$$
\lim _{n \rightarrow \infty} \frac{\phi_{n}^{*}(z) w_{n}^{*}(z)}{\psi_{n, n+k}^{*}(z)}=1
$$

uniformly for $|z| \leq s<1$ and $k$ a fixed integer. As we can see, the right-hand side of (1.3) is well defined even if $\log \mu^{\prime} \in L^{1}$ is not assumed. Hence one may ask whether (1.3) remains true for $\mu^{\prime}$ not satisfying Szegö's condition.

In this paper we obtain the strong and weak asymptotics of the ratio of $\phi_{n}^{*}(z) w_{m}^{*}(z)$ and $\psi_{m, m+n}^{*}(z)$ on $|z| \leq 1$ under the weaker condition $\mu^{\prime}>0$ a.e. in $[0,2 \pi]$.

The main results are stated in $\S 2$. The proofs are given in $\S 3$.

\section{MAIN THEOREMS}

In this section, we only state our main theorems; the proofs will be given in the next section. Our first result will be the strong asymptotic behavior for the ratio of two polynomials. 
Theorem 2.1. If $\mu^{\prime}>0$ a.e. in $[0,2 \pi]$, then

$$
\lim _{n \rightarrow \infty} \frac{\phi_{n}^{*}(z) w_{m}^{*}(z)}{\psi_{m, n+m}^{*}(z)}=1
$$

uniformly for $|z| \leq s<1$, where $m:=m(n) \in \mathbf{N}$ is a function of $n$ and $m \rightarrow \infty$ as $n \rightarrow \infty$.

Remark 1. If $m$ is not a function of $n$, the theorem is still true-it is then a special case of Theorem 1 in [MNT 2].

Remark 2. Relation (1.3) only serves as motivation to study (2.1); it does not reduce to (2.1).

For the $L_{2}$-norm asymptotic, we have the following result.

Theorem 2.2. Assume $\mu^{\prime}>0$ a.e. in $[0,2 \pi]$. Then

$$
\lim _{n \rightarrow \infty} \int_{0}^{2 \pi}|| \frac{\phi_{n}(z) w_{m}(z)}{\psi_{m, m+n}(z)}|-1|^{2} d \theta=0, \quad z=e^{i \theta},
$$

uniformly in $m \geq 1$.

There are several consequences of this result.

Corollary 2.3. Assume $\mu^{\prime}>0$ a.e. in $[0,2 \pi]$. Then

$$
\left.\lim _{n \rightarrow \infty} \int_{0}^{2 \pi}|| \frac{\phi_{n}(z) w_{m}(z)}{\psi_{m, m+n}(z)}\right|^{2}-1 \mid d \theta=0, \quad z=e^{i \theta},
$$

and

$$
\lim _{n \rightarrow \infty} \int_{0}^{2 \pi}|| \frac{w_{m}(z)}{\psi_{m, m+n}(z)}\left|-\frac{1}{\left|\phi_{n}(z)\right|}\right| d \theta=0, \quad z=e^{i \theta},
$$

uniformly in $m \geq 1$.

We give the following weak convergent result to end this section.

Corollary 2.4. Assume $\mu^{\prime}>0$ a.e. in $[0,2 \pi]$. Then

$$
\lim _{n \rightarrow \infty} \int_{0}^{2 \pi} f(\theta)\left|\frac{\phi_{n}(z) w_{m}(z)}{\psi_{m, m+n}(z)}\right|^{2} d \theta=\int_{0}^{2 \pi} f(\theta) d \theta, \quad z=e^{i \theta},
$$

uniformly in $m \geq 1$, for every bounded Borel measurable function $f$ on $[0,2 \pi]$.

\section{Proofs of The THeORems}

Before we prove the results, we first establish some lemmas.

Lemma 3.1 [MNT 1]. Assume $\mu^{\prime}>0$ a.e. in $[0,2 \pi]$, and let $p>0$ and $S$ be real numbers. If

$$
\left(\frac{1}{2 \pi} \int_{0}^{2 \pi}\left(f(\theta) \mu^{\prime}(\theta)\right)^{p} d \theta\right)^{1 / p} \leq S \cdot \frac{1}{2 \pi} \int_{0}^{2 \pi} f(\theta) d \mu(\theta)
$$

holds for every nonnegative continuous $2 \pi$-periodic function $f$. Then $S \geq 1$. 
Lemma 3.2. Let $f$ be a $2 \pi$-periodic continuous function, then

$$
\lim _{n \rightarrow \infty} \int_{0}^{2 \pi} \frac{f(\theta)}{\left|\phi_{n}(z)\right|^{2}} d \theta=\int_{0}^{2 \pi} f(\theta) d \mu(\theta), \quad z=e^{i \theta}
$$

This lemma is a consequence of the following lemma.

Lemma 3.3 [F, p. 198]. Let $n \geq 0$ be an integer. Then

$$
\int_{0}^{2 \pi} \frac{p_{n}(z) \overline{q_{n}(z)}}{\left|\phi_{n}(z)\right|^{2}} d \theta=\int_{0}^{2 \pi} p_{n}(z) \overline{q_{n}(z)} d \mu(\theta)
$$

holds for every $p_{n}$ and $q_{n}$ in $\mathscr{P}_{n}$.

Remark 3. For the varying measures, we have

$$
\int_{0}^{2 \pi} \frac{p_{n}(z) \overline{q_{n}(z)}}{\left|\psi_{m, n}(z)\right|^{2}} d \theta=\int_{0}^{2 \pi} p_{n}(z) \overline{q_{n}(z)} \frac{d \mu(\theta)}{\left|w_{m}(z)\right|^{2}}
$$

for every $p_{n}$ and $q_{n}$ in $\mathscr{P}_{n}$.

Proof of Theorem 2.2. From the remark to Lemma 3.3, we have

$$
\begin{aligned}
\int_{0}^{2 \pi} & || \frac{\phi_{n}(z) w_{m}(z)}{\psi_{m, m+n}(z)}|-1|^{2} d \theta \\
& =\int_{0}^{2 \pi}\left|\frac{\phi_{n}(z) w_{m}(z)}{\psi_{m, m+n}(z)}\right|^{2} d \theta-2 \int_{0}^{2 \pi}\left|\frac{\phi_{n}(z) w_{m}(z)}{\psi_{m, m+n}(z)}\right| d \theta+2 \pi \\
& =\int_{0}^{2 \pi}\left|\phi_{n}(z) w_{m}(z)\right|^{2} \frac{d \mu(\theta)}{\left|w_{m}(z)\right|^{2}}-2 \int_{0}^{2 \pi}\left|\frac{\phi_{n}(z) w_{m}(z)}{\psi_{m, m+n}(z)}\right| d \theta+2 \pi \\
& =4 \pi-2 \int_{0}^{2 \pi}\left|\frac{\phi_{n}(z) w_{m}(z)}{\psi_{m, m+n}(z)}\right| d \theta, \quad z=e^{i \theta} .
\end{aligned}
$$

Therefore, it will be sufficient to prove that

$$
\liminf _{n \rightarrow \infty} \int_{0}^{2 \pi}\left|\frac{\phi_{n}(z) w_{m}(z)}{\psi_{m, m+n}(z)}\right| d \theta \geq 2 \pi
$$

uniformly in $m \geq 1$. To prove this, let $f$ be an arbitrary $2 \pi$-periodic nonnegative continuous function. By Schwarz's inequality, we obtain 


$$
\begin{aligned}
\left(\frac{1}{2 \pi} \int_{0}^{2 \pi}\left(f(\theta) \mu^{\prime}(\theta)\right)^{1 / 4} d \theta\right)^{4} & \left(\frac{1}{2 \pi} \int_{0}^{2 \pi}\left|\frac{\phi_{n}(z) w_{m}(z)}{\psi_{m, m+n}(z)}\right|^{1 / 2}\left(\mu^{\prime}(\theta)\right)^{1 / 4}\left|\frac{\psi_{m, m+n}(z)}{w_{m}(z)}\right|^{1 / 2}\right. \\
& \left.=\left(\frac{1}{2 \pi} \int_{0}^{2 \pi}\left|\frac{\phi_{n}(z) w_{m}(z)}{\psi_{m, m+n}(z)}\right| d \theta\right)^{1 / 4} \frac{1}{\left|\phi_{n}(z)\right|^{1 / 2}} d \theta\right)^{4} \\
& \leq\left(\frac{1}{2 \pi} \int_{0}^{2 \pi} \mu^{\prime}(\theta)\left|\frac{\psi_{m, m+n}(z)}{w_{m}(z)}\right|^{2} d \theta\right) \\
& \leq\left(\frac{1}{2 \pi} \int_{0}^{2 \pi}\left|\frac{\phi_{n}(z) w_{m}(z)}{\psi_{m, m+n}(z)}\right| d \theta\right)^{2}\left(\frac{1}{2 \pi} \int_{0}^{2 \pi}\left|\frac{\psi_{m, m+n}(z)}{w_{m}(z)}\right|^{2 \pi} d \mu(\theta)\right) \\
& \leq\left(\frac{1}{2 \pi} \int_{0}^{2 \pi}\left|\frac{\phi_{n}(z) w_{m}(z)}{\psi_{m, m+n}(z)}\right| d \theta\right)^{2}\left(\frac{1}{2 \pi} \int_{0}^{2 \pi} f(\theta) \frac{1}{\left|\phi_{n}(z)\right|^{2}} d \theta\right), \\
& \left.\left.(z)\right|^{2} d \theta\right)
\end{aligned}
$$

Letting $n \rightarrow \infty$, we obtain by Lemma 3.2 that

$$
\begin{aligned}
& \left(\frac{1}{2 \pi} \int_{0}^{2 \pi}\left(f(\theta) \mu^{\prime}(\theta)\right)^{1 / 4} d \theta\right)^{4} \\
& \quad \leq\left(\liminf _{n \rightarrow \infty} \frac{1}{2 \pi} \int_{0}^{2 \pi}\left|\frac{\phi_{n}(z) w_{m}(z)}{\psi_{m, m+n}(z)}\right| d \theta\right)^{2}\left(\frac{1}{2 \pi} \int_{0}^{2 \pi} f(\theta) d \mu(\theta)\right),
\end{aligned}
$$

$z=e^{i \theta}$, for any $m \geq 1$. Since $f$ was an arbitrary $2 \pi$-periodic nonnegative continuous function and the limit is uniform in $m \geq 1$, this implies (3.1) according to Lemma 3.1. The proof of the theorem is complete.

Proof of Corollary 2.3. First we prove (2.2). Notice that according to Schwarz's inequality, we have

$$
\begin{aligned}
& \left(\left.\int_{0}^{2 \pi}|| \frac{\phi_{n}(z) w_{m}(z)}{\psi_{m, m+n}(z)}\right|^{2}-1 \mid d \theta\right)^{2} \\
& \quad=\left(\int_{0}^{2 \pi}|| \frac{\phi_{n}(z) w_{m}(z)}{\psi_{m, m+n}(z)}|-1||| \frac{\phi_{n}(z) w_{m}(z)}{\psi_{m, m+n}(z)}|+1| d \theta\right)^{2} \\
& \quad \leq\left(\int_{0}^{2 \pi}|| \frac{\phi_{n}(z) w_{m}(z)}{\psi_{m, m+n}(z)}|-1|^{2} d \theta\right)\left(\int_{0}^{2 \pi}|| \frac{\phi_{n}(z) w_{m}(z)}{\psi_{m, m+n}(z)}|+1|^{2} d \theta\right),
\end{aligned}
$$


$z=e^{i \theta}$. By Theorem 2.2, the first integral on the right tends to zero uniformly in $m \geq 1$ as $n \rightarrow \infty$. As for the second integral,

$$
\begin{aligned}
\int_{0}^{2 \pi} & || \frac{\phi_{n}(z) w_{m}(z)}{\psi_{m, m+n}(z)}|+1|^{2} d \theta \\
& =\int_{0}^{2 \pi}\left|\frac{\phi_{n}(z) w_{m}(z)}{\psi_{m, m+n}(z)}\right|^{2} d \theta+2 \int_{0}^{2 \pi}\left|\frac{\phi_{n}(z) w_{m}(z)}{\psi_{m, m+n}(z)}\right| d \theta+2 \pi \\
& \leq \int_{0}^{2 \pi}\left|\frac{\phi_{n}(z) w_{m}(z)}{\psi_{m, m+n}(z)}\right|^{2} d \theta+2\left(2 \pi \int_{0}^{2 \pi}\left|\frac{\phi_{n}(z) w_{m}(z)}{\psi_{m, m+n}(z)}\right|^{2} d \theta\right)^{1 / 2}+2 \pi \\
& =\int_{0}^{2 \pi}\left|\phi_{n}(z)\right|^{2} d \mu(\theta)+2\left(2 \pi \int_{0}^{2 \pi}\left|\phi_{n}(z)\right|^{2} d \mu(\theta)\right)^{1 / 2}+2 \pi \leq 8 \pi,
\end{aligned}
$$

$z=e^{i \theta}$, where the first inequality follows from Schwarz's inequality and the last equality follows from the remark to Lemma 3.3. Thus (2.2) holds.

Next we prove (2.3). By Schwarz's inequality, we have

$$
\begin{aligned}
\left(\int_{0}^{2 \pi}\right. & \left.|| \frac{w_{m}(z)}{\psi_{m, m+n}(z)}\left|-\frac{1}{\left|\phi_{n}(z)\right|}\right| d \theta\right)^{2} \\
\quad & \left(\int_{0}^{2 \pi}|| \frac{w_{m}(z) \phi_{n}(z)}{\psi_{m, m+n}(z)}|-1| \frac{1}{\left|\phi_{n}(z)\right|} d \theta\right)^{2} \\
& \leq \int_{0}^{2 \pi}|| \frac{w_{m}(z) \phi_{n}(z)}{\psi_{m, m+n}(z)}|-1|^{2} d \theta \cdot \int_{0}^{2 \pi} \frac{1}{\left|\phi_{n}(z)\right|^{2}} d \theta, \quad z=e^{i \theta} .
\end{aligned}
$$

The second integral on the right-hand side equals $\int_{0}^{2 \pi} d \mu(\theta)$ by Lemma 3.3, and by Theorem 2.2 the first term tends to zero uniformly in $m \geq 1$ as $n \rightarrow \infty$. Thus (2.3) follows. The proof of Corollary 2.3 is complete.

Proof of Corollary 2.4. The result is a direct consequence of (2.2).

Proof of Theorem 2.1. For $m=m(n)$, we first prove that

$$
\liminf _{n \rightarrow \infty} \frac{\phi_{n}^{*}(0) w_{m}^{*}(0)}{\psi_{m, m+n}^{*}(0)} \geq 1
$$

In fact, from Schwarz's inequality, we have (notice that $\left|p_{n}^{*}(z)\right|=\left|p_{n}(z)\right|$ for $p_{n} \in \mathscr{P}_{n}$ when $\left.|z|=1\right)$ 


$$
\begin{aligned}
& \left.\int_{0}^{2 \pi}|| \frac{\psi_{m, m+n}^{*}(z)}{\phi_{n}^{*}(z) w_{m}^{*}(z)}\right|^{1 / 2}-1 \mid\left(\mu^{\prime}(\theta)\right)^{1 / 4} d \theta \\
& =\left.\int_{0}^{2 \pi}|| \frac{\phi_{n}^{*}(z) w_{m}^{*}(z)}{\psi_{m, m+n}^{*}(z)}\right|^{1 / 2}-\left.1|| \frac{\psi_{m, m+n}^{*}(z)}{w_{m}^{*}(z)}\right|^{1 / 2}\left(\mu^{\prime}(\theta)\right)^{1 / 4} \frac{1}{\left|\phi_{n}^{*}(z)\right|^{1 / 2}} d \theta \\
& \leq\left(\left.\int_{0}^{2 \pi}|| \frac{\phi_{n}^{*}(z) w_{m}^{*}(z)}{\psi_{m, m+n}^{*}(z)}\right|^{1 / 2}-1 \mid d \theta\right)^{1 / 2} \\
& \times\left(\int_{0}^{2 \pi}\left|\frac{\psi_{m, m+n}^{*}(z)}{w_{m}^{*}(z)}\right|^{2} \mu^{\prime}(\theta) d \theta\right)^{1 / 4}\left(\int_{0}^{2 \pi} \frac{1}{\left|\phi_{n}^{*}(z)\right|^{2}} d \theta\right)^{1 / 4} \\
& \leq\left(\left.\int_{0}^{2 \pi}|| \frac{\phi_{n}^{*}(z) w_{m}^{*}(z)}{\psi_{m, m+n(z)}^{*}(z)}\right|^{1 / 2}-1 \mid d \theta\right)^{1 / 2}\left(\int_{0}^{2 \pi} d \mu(\theta)\right)^{1 / 4} \\
& \leq\left(\left.\int_{0}^{2 \pi}|| \frac{\phi_{n}^{*}(z) w_{m}^{*}(z)}{\psi_{m, m+n}^{*}(z)}\right|^{1 / 2}-1||\left|\frac{\phi_{n}^{*}(z) w_{m}^{*}(z)}{\psi_{m, m+n}^{*}(z)}\right|^{1 / 2}+1 \mid d \theta\right)^{1 / 2} \\
& \times\left(\int_{0}^{2 \pi} d \mu(\theta)\right)^{1 / 4} \\
& =\left(\int_{0}^{2 \pi}|| \frac{\phi_{n}^{*}(z) w_{m}^{*}(z)}{\psi_{m, m+n}^{*}(z)}|-1| d \theta\right)^{1 / 2}\left(\int_{0}^{2 \pi} d \mu(\theta)\right)^{1 / 4} \\
& \leq\left(2 \pi \int_{0}^{2 \pi}|| \frac{\phi_{n}^{*}(z) w_{m}^{*}(z)}{\psi_{m, m+n}^{*}(z)}|-1|^{2} d \theta\right)^{1 / 4}\left(\int_{0}^{2 \pi} d \mu(\theta)\right)^{1 / 4}, \quad z=e^{i \theta} .
\end{aligned}
$$

Using Theorem 2.2, we obtain

$$
\left.\lim _{n \rightarrow \infty} \int_{0}^{2 \pi}|| \frac{\psi_{m, m+n}^{*}(z)}{\phi_{n}^{*}(z) w_{m}^{*}(z)}\right|^{1 / 2}-1 \mid\left(\mu^{\prime}(\theta)\right)^{1 / 4} d \theta=0, \quad z=e^{i \theta} .
$$

uniformly in $m \geq 1$.

From this we claim that, for $m=m(n)$,

$$
\limsup _{n \rightarrow \infty}\left|\frac{\psi_{m, m+n}^{*}\left(e^{i \theta}\right)}{\phi_{n}^{*}\left(e^{i \theta}\right) w_{m}^{*}\left(e^{i \theta}\right)}\right|^{1 / 2} \leq 1, \quad \theta \text { a.e. in }[0,2 \pi] .
$$

Assume on the contrary that there exists an $E \subset[0,2 \pi],|E|>0$, such that

$$
\limsup _{n \rightarrow \infty}\left|\frac{\psi_{m, m+n}^{*}\left(e^{i \theta}\right)}{\phi_{n}^{*}\left(e^{i \theta}\right) w_{m}^{*}\left(e^{i \theta}\right)}\right|^{1 / 2}>1+\delta, \quad \theta \text { a.e. in } E,
$$

where $\delta<0$. Thus we can find a subsequence $n_{k}$ of $\mathbf{N}$ such that

$$
\lim _{n_{k} \rightarrow \infty}\left(\left|\frac{\psi_{m, m+n_{k}}^{*}\left(e^{i \theta}\right)}{\phi_{n_{k}}^{*}\left(e^{i \theta}\right) w_{m}^{*}\left(e^{i \theta}\right)}\right|^{1 / 2}-1\right)>\delta, \quad \theta \text { a.e. in } E,
$$


for $m=m\left(n_{k}\right)$. From this, we have, for $m=m\left(n_{k}\right)$,

$$
\begin{aligned}
& \left.\lim _{n_{k} \rightarrow \infty} \int_{0}^{2 \pi}|| \frac{\psi_{m, m+n_{k}}^{*}(z)}{\phi_{n_{k}}^{*}(z) w_{m}^{*}(z)}\right|^{1 / 2}-1 \mid\left(\mu^{\prime}(\theta)\right)^{1 / 4} d \theta \\
& \quad \geq\left.\lim _{n_{k} \rightarrow \infty} \int_{E}|| \frac{\psi_{m, m+n_{k}}^{*}(z)}{\phi_{n_{k}}^{*}(z) w_{m}^{*}(z)}\right|^{1 / 2}-1 \mid\left(\mu^{\prime}(\theta)\right)^{1 / 4} d \theta \\
& \quad \geq\left.\int_{E} \liminf _{n_{k} \rightarrow \infty}|| \frac{\psi_{m, m+n_{k}}^{*}(z)}{\phi_{n_{k}}^{*}(z) w_{m}^{*}(z)}\right|^{1 / 2}-1 \mid\left(\mu^{\prime}(\theta)\right)^{1 / 4} d \theta \\
& \geq \int_{E} \delta\left(\mu^{\prime}(\theta)\right)^{1 / 4} d \theta>0, \quad z=e^{i \theta},
\end{aligned}
$$

since $\mu^{\prime}>0$ a.e. in $E$. This contradicts (3.3), so we have (3.4). Inequality (3.4) implies

$$
\limsup _{n \rightarrow \infty}\left|\frac{\psi_{m, n+n}^{*}\left(e^{i \theta}\right)}{\phi_{n}^{*}\left(e^{i \theta}\right) w_{m}^{*}\left(e^{i \theta}\right)}\right| \leq 1, \quad \theta \text { a.e. in }[0,2 \pi] .
$$

Since $\psi_{m, m+n}^{*}(z) / \phi_{n}^{*}(z) w_{m}^{*}(z)$ (the zeros of $\phi_{n}^{*}(z)$ and $w_{n}^{*}(z)$ lie in $|z|>1$ ) is analytic in $|z| \leq 1$, then

$$
\begin{aligned}
\frac{\psi_{m, m+n}^{*}(0)}{\phi_{n}^{*}(0) w_{m}^{*}(0)} & =\frac{1}{2 \pi} \int_{0}^{2 \pi} \frac{\psi_{m, m+n}^{*}(z)}{\phi_{n}^{*}(z) w_{m}^{*}(z)} d \theta \\
& \leq \frac{1}{2 \pi} \int_{0}^{2 \pi}\left|\frac{\psi_{m, m+n}^{*}(z)}{\phi_{n}^{*}(z) w_{m}^{*}(z)}\right| d \theta, \quad z=e^{i \theta}
\end{aligned}
$$

Then, from (3.5), we have

$$
\begin{aligned}
\limsup _{n \rightarrow \infty} & \frac{\psi_{m, m+n}^{*}(0)}{\phi_{n}^{*}(0) w_{m}^{*}(0)} \\
\quad \leq & \limsup _{n \rightarrow \infty} \frac{1}{2 \pi} \int_{0}^{2 \pi}\left|\frac{\psi_{m, m+n}^{*}(z)}{\phi_{n}^{*}(z) w_{m}^{*}(z)}\right| d \theta \\
\quad \leq & \frac{1}{2 \pi} \int_{0}^{2 \pi} \limsup _{n \rightarrow \infty}\left|\frac{\psi_{m, m+n}^{*}(z)}{\phi_{n}^{*}(z) w_{m}^{*}(z)}\right| d \theta \leq 1, \quad z=e^{i \theta} .
\end{aligned}
$$

Thus

$$
\liminf _{n \rightarrow \infty} \frac{\phi_{n}^{*}(0) w_{m}^{*}(0)}{\psi_{m, m+n}^{*}(0)}=1 / \limsup _{n \rightarrow \infty} \frac{\psi_{m, m+n}^{*}(0)}{\phi_{n}^{*}(0) w_{m}^{*}(0)} \geq 1 .
$$

This completes the proof of (3.2).

Now we can turn our attention to (2.1). First let us show that, for $m=m(n)$, $\left\{\phi_{n}^{*}(z) w_{m}^{*}(z) / \psi_{m, m+n}^{*}(z)\right\}, n \in \mathbf{N}$, is uniformly bounded on each compact subset of $|z|<1$. In fact, using Cauchy's formula and Cauchy-Schwarz's inequality, we obtain

$$
\begin{aligned}
& \left|\frac{\phi_{n}^{*}(z) w_{m}^{*}(z)}{\psi_{m, m+n}^{*}(z)}\right| \leq \frac{1}{2 \pi} \int_{0}^{2 \pi}\left|\frac{u \phi_{n}^{*}(u) w_{m}^{*}(u)}{\psi_{m, m+n}^{*}(u)(u-z)}\right| d \theta \\
& \quad \leq\left(\frac{1}{2 \pi} \int_{0}^{2 \pi}\left|\frac{u}{(u-z)}\right|^{2} d \theta\right)^{1 / 2}\left(\frac{1}{2 \pi} \int_{0}^{2 \pi}\left|\frac{\phi_{n}^{*}(u) w_{m}^{*}(u)}{\psi_{m, m+n}^{*}(u)}\right|^{2} d \theta\right)^{1 / 2} \\
& \leq \frac{1}{\inf \{|z-u|:|u|=1\}}, \quad u=e^{i \theta} .
\end{aligned}
$$


Since $\phi_{n}^{*}(z) w_{m}^{*}(z) / \psi_{m, m+n}^{*}(z)$ is analytic in $|z|<1$, then it is an normal family. Take, in $|z|<1,\left\{\phi_{n}^{*}(z) w_{m}^{*}(z) / \psi_{m, m+n}^{*}(z)\right\}, n \in \Gamma, \Gamma \subset \mathbf{N}$, a convergent subsequence whose limit is $S_{\Gamma}$. It is sufficient to prove that any $S_{\Gamma}=1$. To this end, we only need to prove that

$$
\mathfrak{R}\left\{\log S_{\Gamma}(z)\right\}=\log \left|S_{\Gamma}(z)\right|=0 .
$$

However, for the Poisson's kernel $P(u, z)$, using Jensen's inequality and Corollary 2.4 , we get

$$
\begin{aligned}
\left|S_{\Gamma}(z)\right|^{2} & =\lim _{n \in \Gamma}\left|\frac{\phi_{n}^{*}(z) w_{m}^{*}(z)}{\psi_{m, m+n}^{*}(z)}\right|^{2} \\
& =\lim _{n \in \Gamma} \exp \left(\frac{1}{2 \pi} \int_{0}^{2 \pi} P(z, u) \log \left|\frac{\phi_{n}^{*}(u) w_{m}^{*}(u)}{\psi_{m, m+n}^{*}(u)}\right|^{2} d \theta\right) \\
& \leq \lim _{n \in \Gamma} \frac{1}{2 \pi} \int_{0}^{2 \pi} P(z, u)\left|\frac{\phi_{n}^{*}(u) w_{m}^{*}(u)}{\psi_{m, m+n}^{*}(u)}\right|^{2} d \theta \\
& =\frac{1}{2 \pi} \int_{0}^{2 \pi} P(z, u) d \theta=1, \quad u=e^{i \theta .}
\end{aligned}
$$

Hence,

$$
\mathfrak{R}\left\{\log S_{\Gamma}(z)\right\}=\log \left|S_{\Gamma}(z)\right| \leq 0 .
$$

But according to (3.2), we have

$$
\mathfrak{R}\left\{\log S_{\Gamma}(0)\right\} \geq 0 .
$$

Therefore, using the maximum principle for harmonic functions, it follows that

$$
\mathfrak{R}\left\{\log S_{\Gamma}(z)\right\}=0 \text {. }
$$

\section{REFERENCES}

[F] G. Freud, Orthogonal polynomials, Pergamon Press, New York, 1971.

[G] Ya. L. Geronimus, Polynomials orthogonal on a circle and interval, Pergamon Press, Oxford, 1960.

[LP] X. Li and K. Pan, Strong and weak convergence of rational functions orthogonal on the unit circle, submitted..

[Lo1] G. López, Szegö's theorem for polynomials orthogonal with respect to varying measures, Orthogonal Polynomials and their Applications (M. Alfaro et al., eds.), Lecture Notes in Math., vol. 1329, Springer-Verlag, Berlin, 1988, pp. 255-260.

[Lo2] _ - On the asymptotics of the ratio of orthogonal polynomials and the convergence of multipoint Padé approximants, Math. USSR-Sb. 56 (1987), 207-219.

[Lo3] _ Asymptotics of polynomials orthogonal with respect to varying measures, Constr. Approx. 5 (1989), 199-219.

[L04] _ Convergence of Padé approximants of Stieltjes type meromorphic functions and comparative asymptotics for orthogonal polynomials, Math. USSR-Sb 64 (1989), 207-227.

[MNT1] A. Máté, P. Nevai and V. Totik, Strong and weak convergence of orthogonal polynomials, Amer. J. Math. 109 (1987), 239-282.

[MNT2] _ Extensions of Szegö's theory of orthogonal polynomials, II, Constr. Approx. 3 (1987), 51-72. 
[P1] K. Pan, Strong and weak convergence of orthogonal systems of rational functions on the unit circle, J. Comput. Appl. Math. 46 (1993), 427-436.

[P2] On the convergence of rational interpolation approximant of Caratheodory functions, J. Comput. Appl. Math. (to appear).

Department of Mathematics and Computer Science, Barry University, Miami Shores, FL 33161

E-mail address: pan@buvax.barry.edu 